\title{
A Review on Synthesis of CNTs and its Application in Conductive Paints
}

\author{
Ravuluri Sahithi $^{1}$, Bajpai Harshit ${ }^{1}$, Khandelwal Mansi ${ }^{1}$, Bajad Ganesh ${ }^{1}$, Vijayakumar R.P. ${ }^{1}$ \\ Visvesvaraya National Institute of Technology, Nagpur, Maharashtra ${ }^{1}$
}

\begin{abstract}
Solar paints or conducting paints have replaced the solar panels as it requires high installation and maintenance costs. Uses of cadmium and other nano particles as fillers to efficiently conduct solar energy have been extensively reported in the literature. However, these nanoparticles have adverse effect on the environment. In the present work, literature on CNT synthesis and its use as fillers to induce conductive properties on paints is discussed in details.
\end{abstract}

\section{Keywords: Solar paints; CNTs; Polymers; CVD}

\section{INTRODUCTION}

Conductive paints are prepared by dispersing the material which can induce conductive property in the paint matrix. The conductive paint should be economical, environmental friendly and efficiently absorb the sunlight. The economic feasibility of solar energy utilization depends upon efficient collection, conversion and storage. The efficient utilization of solar energy for various process applications requires the use of the collector systems which first capture as much as possible of incoming radiation and deliver a high fraction of the captured energy for propagation. The conversion efficiency of a collector system is limited by the thermal losses from the heated absorber due to conduction, convection and radiation. The losses become increasingly significant at higher temperatures. Thus the effective utilization of solar radiation can be achieved by an efficient and low cost 'solar selective coating' [1].

The solar paints prepared using graphene and other materials which can absorb sunlight were reported by Matthew et al., [2]. Nanoparticles like cadmium were used as fillers for the preparation of conducting solar paints. However, such solar paint cannot be applied on all solid state devices [2]. Zhu et al., [3] reported that the copper nanoparticles filled CNTs may be used as fillers.

CNTs are long cylinders of covalently bonded carbon atoms which possess extraordinary electronic and mechanical properties. CNTs are available in the form of Single and multi walled structures. Single-wall carbon nanotubes (SWCNTs) which are the fundamental cylindrical structure and multi-wall carbon nanotubes (MWCNTs) which are made of coaxial cylinders, having interlayer spacing close to that of the interlayer distance in graphite $(0.34 \mathrm{~nm})$. These cylindrical structures are only few nanometres in diameter, but length wise it can be tens of microns long with most end capped with half of a fullerene molecule [4].

The main objective of the present study is to understand the techniques of CNT synthesis and its dispersion techniques in the paint matrix to obtain conductive paints.

\section{SYNTHESIS OF CARBON NANOTUBES}

CNTs are prepared by three different methods such as arc discharge (C60 fullerenes are produced by this method

which is perhaps the easiest way to produce CNTs), laser ablation (an intense laser pulse is targeted to vaporize a carbon rod in presence of an inert gas. This method mainly produces single-walled CNTs) and chemical vapour deposition (CVD) method. CVD technique is widely used as it is carried out at a much lower reaction temperature and also good control over length and structure of the nanotubes is attainable. It also offers the advantage to adjust the reaction conditions to produce the desired CNTs (single-walled or multi-walled). CVD can be carried out with or without the presence of catalyst [4].

The techniques of CVD are discussed below in detail.

\section{ChemiCAL VAPOUR DEPOSITION TECHNIQUe}

\section{A. Catalytic chemical vapour deposition}

In this technique, a mixture of hydrocarbons, metal catalyst along with inert gas is introduced into the reaction chamber. At around $700-900{ }^{\circ} \mathrm{C}$ and atmospheric pressure, nanotubes form on the catalyst substrate by the decomposition of the hydrocarbon [4-11]. These CNTs can be collected upon cooling the system to room temperature. In the case of a liquid hydrocarbon, liquid is heated in a flask and an inert gas (usually argon) is purged through it which in turn carries the hydrocarbon vapours into the reaction zone. In case of solid hydrocarbons, it can be kept in the low temperature zone of the reaction tube. The volatile materials directly turn from solid to vapour and perform CVD while passing over the catalyst kept in the high temperature zone. Like CNT precursors, catalyst used in CVD can also be in any form (solid, liquid or gas) which may be suitably placed in the reactor or externally fed. Alternatively, catalyst coated substrates can be used to catalyse the CNT growth. The size of the catalyst metal particles used in this technique has influence on the diameter of the nanotubes. Two growth mechanisms can be expected from this technique (Tip growth and Base growth) depending upon the adhesion between the catalyst particles and the substrate (if it is weak, tip growth and if it is strong, base growth). It is well known that hydrocarbons can be easily broken at high temperatures (pyrolysis), but in the presence of suitable metal catalyst hydrocarbons can be decomposed at lower temperatures (catalytic pyrolysis). 
The key of CNT growth by CVD is to achieve functionalization is associated with a change of hydrocarbon decomposition on the metal surface alone and hybridization from $\operatorname{sp} 2$ to $\operatorname{sp} 3$ and also the loss of $\pi$ prohibit spontaneous aerial pyrolysis and this is done by the proper selection of catalyst and hydrocarbon materials, vapor pressure of hydrocarbon, concentration of the catalyst, the CVD reaction temperature [12].

For the use of CNTs to induce conductive properties in paints, CNTs are to be made conductive. This can be done by incorporating metallic conductive nanoparticles into the CNTs in a separate functionalization step (endohedral functionalization) or by directly using the metallic particles as a catalyst during the preparation of CNTs using CVD [13, 14]. Zhu et al. [3] reported the formation of bamboo-like CNTs using a copper foil by CVD from ethanol. They found that the yield and size of CNTs increases with increasing the temperature. The CNTs which were prepared at around $700^{\circ} \mathrm{C}$ had copper droplet tip and those at $800-900^{\circ} \mathrm{C}$ had a copper nanoparticle inside. They also observed that the enhancement of CNT growth with increase in the duration up to 30 minutes and prolonged duration up to 60 minutes did not shown any increase in the yield of CNTs to an appreciable level. It was observed and proposed that a carbon film first deposits on the top surface of the copper foil while the top surface of the copper foil partially melted and migrated across the carbon film where CNTs are formed [3].

\section{B. Non - Catalytic Chemical Vapour Deposition}

This method is a modification of the above technique wherein uniform, well-aligned multi walled CNTs (MWCNTs) grow in the channels of alumina porous oxide films. These alumina templates are commercially available or can be prepared in the laboratory by anodic oxidation of aluminium. By changing the process parameters, required thickness and pore diameters of the alumina films can be obtained which in turn affects the growth of CNTs in the pores. At high temperature (generally $700-900{ }^{\circ} \mathrm{C}$ ), on passing hydrocarbon gas into the tubular reactor which has an alumina template inside it, carbon deposits on the inner walls of the alumina template. After this process, the CNT filled template is taken out of the tubular reactor and washed with hydrofluoric acid to separate the CNTs from the template. The resulting nanotubes are straight and have uniform diameter and thickness (thickness depends on the length of the deposition process) [15].

\section{IV.FunCTIONALIZATION OF CNTS}

The performance of CNT depends on its dispersion in the matrix and the interfacial interaction between the CNT and the matrix. The carbon atoms on the CNT walls are chemically stable because of the aromatic nature of the bond and as a result the reinforcing CNTs are inert and can interact with the surrounding matrix mainly through the van der waal forces of attraction. Hence the efficient load transfer is not possible across the CNT-matrix interface. It can be attained by the modification in the surface properties of the CNTs by either chemical or physical functionalization $[4,16]$

A. Chemical functionalization

It is based on the covalent linkage of functional entities and the carbon of CNTs. Direct covalent sidewall theaction with some molecules of high chemical activity such as fluorine. It was reported that the fluorination of purified SWCNTs occurs at the temperatures upto $325^{\circ} \mathrm{C}$ and the process is reversible using anhydrous hydrazine which can remove the fluorine. The fluorinated CNTs have C-F bonds that are weaker than those in alkyl fluorides and thus providing substitution sites for additional functionalization. Successful replacement of the fluorine atoms by amino, alkyl and hydroxyl groups has been reported [16].

Defect functionalization is another method for covalent functionalization of CNTs. An oxidative process using strong acids (such as nitric acid) or by using strong oxidants (such as $\mathrm{KMnO} 4$ ) can induce defects on the side walls as well as open ends of the CNTs. These defects are stabilized by bonding with carboxylic acid or hydroxyl groups. These functional groups have rich chemistry and the CNTs can be used as precursors for further chemical reactions. The chemically functionalized CNTs have strong interfacial bonds with induced high mechanical and functional properties. However this method has few drawbacks, firstly, during the functionalization reaction, (especially in the ultrasonication process), a large number of defects are created on the sidewalls of CNTs and in some extreme cases CNTs are fragmented into smaller pieces. This leads to decrease in the mechanical properties of CNTs as well as disruption of $\pi$ electron system in the nanotubes. Secondly, the concentrated acids or oxidants used in covalent functionalization are not environment friendly [17].

\section{B. Physical functionalization}

Physical functionalization has been put forward as an alternative method for tuning the interfacial properties of nanotubes.

The physical functionalization techniques reported by Korneva[15] are discussed below,

1] Polymer wrapping:

The suspension of CNTs in the presence of polymers leads to the wrapping of the polymer around the CNTs to form super molecular complexes of CNTs. This process is achieved through the Van der Waals interactions and $\pi-\pi$ stacking between CNTs and polymer chains containing aromatic rings.

2] Surfactant adsorption:

Polymers surfactants have also been employed to functionalize CNTs. The effects of different types of surfactants (non-ionic, anionic and cationic) were studied. The physical adsorption of the surfactant on CNT surface lowered the surface tension of the CNT, effectively preventing the formation of aggregates and hence improving the dispersion. Moreover the surfactant treated CNTs overcome the Van der Waals attraction by electrostatic or stearic repulsive forces. The efficiency of this method depends on the properties of surfactants, medium chemistry and the base matrix. It was reported that cationic surfactants are favourable for water soluble 
polymers and for the water insoluble polymers CNT dispersion was promoted by non-ionic surfactant. The treatment of non-ionic surfactants is based on strong hydrophobic attraction between the solid surface and the tail group of surfactant. Once the surfactant is adsorbed onto the filler surface, the surfactant molecules are self assembled into miscelles above a critical miscelle concentration (CMC) [16].

\section{3] Endohedral method}

In this method guest atoms or molecules are stored in the inner cavity of the CNTs by the capillary effect. The incorporation of guest atoms takes place at defect sites localized at the ends or on the sidewalls. The combination of these two materials (CNTs and guest molecules) is particularly useful to integrate the properties of the two components in hybrid materials for use in applications such as catalysis, energy storage, nanotechnology and molecular scale devices. This filling of carbon nanotubes can be done in two ways:

Filling by exploiting the phenomenon of spontaneous penetration:

Capillary absorption is caused by extra pressure given by the laplace equation of capillarity.

$P_{a}-P_{m}=\frac{2 \gamma \cos \theta}{R}$

Where,

$\mathrm{P}_{\mathrm{a}}$ - Atmospheric pressure,

$\mathrm{P}_{\mathrm{m}}$ - Pressure under the meniscus,

$\gamma$ - Surface tension at the liquid air interface,

$\Theta$ - Liquid solid contact angle and

$\mathrm{R}$ - Inner radius of the nanotube

From Laplace equation, we see that the difference ( $\mathrm{Pa}-$ Pm) will be positive whenever $\theta$ is less than $90^{\circ}$ and negative when vice versa.

Since the meniscus forms spontaneously, this extra pressure will pull the liquid into the nanotube if $(\mathrm{Pa}-\mathrm{Pm})$ is greater than zero, provided the pressure in the reservoir is atmospheric. In short, to fill the nanotubes with some liquid, the contact angle must be less than $90{ }^{\circ}$. If the contact angle is greater than $90^{\circ}$, extra pressure must be applied to the liquid in order to impregnate the nanotube. The contact angle is related to the surface energies of the constituent materials as

$\cos \theta=\frac{\gamma_{s v}-\gamma_{s l}}{\gamma_{l v}}$

Where,

$\gamma$ represents surface energy and subscripts sv, sl and lv represent solid-vapour, solid-liquid and liquid-vapour respectively.

For liquids, the surface energy is exactly the surface tension i.e., the spontaneous force acting at the imaginary cut to resist the surface extinction. It was reported that metal oxides and metal carbides fill the nanotubes and not the pure metals. The reason behind it is that the carbides and oxides of metals have a surface tension lower than the surface tension of pure metals. So it was assumed that the reported filling of nanotubes with metals infact is the filling of the nanotubes with metal oxides or metal carbides. [17] Benjamin et al. [17] reported that the liquids with surface tension less than $180 \mathrm{mN} / \mathrm{M}$ can moisten the inner cavity of a nanotube at atmospheric pressure.

Alternatively, in the wet chemistry method, the nanotubes were first filled with metals salts and heated in a furnace. The temperature of the furnace depends on the type of metal salt used. The metal salts decompose into their respective elements at suitable temperatures. It was found that $70 \%$ of the resultant nanotubes were filled, with a very small amount of nanoparticles [15].

\section{DISPERSION OF CNTS}

The commercially available CNTs are usually in the form of entangled bundles, which gives difficulties in dispersion. Dispersion of CNTs is not only a geometrical problem (dealing with the length and size of the CNTs) but also relates to a method on how to separate individual CNTs from CNT agglomerates and stabilize them in polymer matrix to avoid secondary agglomeration [16]. Incorporation of CNTs into a matrix requires exceptionally large quantity of particles. The high aspect ratio of fillers gives difficulties in uniform dispersion of these particles [18]. Thus the proper dispersion method to be adopted which depends upon the matrix in which the CNTs are to be dispersed. The extent of dispersion may be obtained by using Raman spectroscopy, X-ray diffraction studies etc [16].

The different dispersion techniques suggested by Peng et. al.[16] are ultrasonication, calendaring, ball milling, stirring and extrusion techniques. Ultrasonication is the act of using ultrasound energy to agitate particles in a solution. It is usually achieved by using an ultrasonic bath or an ultrasonic probe. The principle behind ultrasonication is that when ultrasound waves propagate through series of compression, attenuated waves are induced in the medium through which the waves are passed. The production of such shock waves promotes the peeling of individual nanoparticles (mostly peel off the nanoparticles located at the outer part of the bundle) and thus results in the separation of individual nanoparticles from bundles. Sonication at higher rate to be avoided as it may lead to the damage of CNTs. This method is quite suitable to disperse CNTs in liquids having low viscosity. Calendaring technique is generally used when the dispersion matrix is viscous. In this method, high shear stresses are applied to disentangle CNT bundles and to distribute them into the matrix, while a short residence time will limit the breakage of individual nanotubes. However, there are several concerns in using calendaring technique for CNT dispersion. For example, generally the minimum gap between the rollers is maintained about 1$5 \mu \mathrm{m}$, which is comparable to the lengths of CNTs, but is much larger than the diameter of individual CNTs. Such dimensional disparities between the roller gap and the dimensions of CNT may suggest that calendaring can better disperse the large agglomerated CNTs into smaller agglomerates at sub micron level (although some individual CNTs may be disentangled out from the agglomerates). 
Ball milling is a grinding technique which is used to grind The process of preparation of vertically aligned carbon materials into extremely fine powder for use in paints, nanotubes that are completely encapsulated by a dense ceramics etc. In this technique, a high pressure is network of copper nanoparticles involves the conformal generated locally because of the collisions between tiny deposition of pyrolytic carbon. These stabilized arrays can and rigid balls in a concealed container. In stirring be functionalized using oxygen plasma treatment (to technique the size, shape and speed of the propeller improve wettability) and then are infiltrated with an controls dispersion results. In this technique CNTs tend to re-agglomerate as they are only separated but not stabilized during separation. The extrusion process consists of twin screws which rotate at high speed and creates a high shear forces which helps in dispersion. This technique is particularly used for producing CNT nano composites with high filler content [16].

\section{CONDUCTING PAINTS WiTh CNT}

The procedure for the preparation of conductive paints with CNT is discussed as below.

To prepare a conductive paint (with CNTs as fillers), firstly CNTs are to be synthesized by chemical vapour deposition technique with the incorporation of copper nanoparticles. The addition of nano particles in CNTs is obtained by using a copper foil during the process of CNT synthesis [3].Powdered form of CNTs are not preferred as it leads to health issues [19]. The as prepared CNTs are to be dispersed properly in a suitable solvent (using ultrasonicator). The solvent should be compatible with paint constituents and also should be environment friendly. The rate of ultrasonication to be controlled in such a way that the aspect ratio of CNTs (which give unique properties to it) should not be reduced due to the shear forces applied on CNTs. The prepared nano fluid having CNT particles is to be properly mixed with paint (using ultrasonicator). With variation in volume fraction of CNTs in the paint matrix, its conductivity varies. Conductivity for the different volume fraction of CNTs are to be checked and the volume fractions to be optimized.

In another method, CNTs are to be prepared using suitable catalyst by CVD method and then obtained CNTs are to be functionalized using metal oxides (copper oxide) using endohedral method of functionalization. During this functionalization step the metal oxide particles get filled in CNTs by capillary action (initially metal oxide particles are to be dispersed in suitable solvent then CNTs are to be added with suitable pressure so that the particles get incorporated in the CNTs through capillary action). Then these functionalized CNTs are to be dispersed in suitable solvent using ultrasonicator. The extent of dispersion may be analysed using Raman spectroscopy and the efficiency of prepared paint need to be studied.

The methods of preparation of copper nanoparticles filled CNTs are reported in literature. Copper nano particle filled multiwalled carbon nanotubes can be synthesized by chemical reduction method using chemical vapour deposition technique and copper chloride. $\mathrm{Cu}$ /MWCNTs based nanofluids are synthesized by dispersing nanocomposites of $\mathrm{Cu} / \mathrm{MWCNT}$ in deionized water and ethylene glycol. A maximum thermal conductivity at a very low volume fraction of $\mathrm{Cu} / \mathrm{MWCNT}$ s can be obtained by homogeneous dispersion of $\mathrm{Cu}$ on the MWCNTs [20]. aqueous, supersaturated $\mathrm{Cu}$ salt solution. After drying, the salt forms a stabilizing crystal network throughout the array. The calcination and $\mathrm{H}_{2}$ reduction, leads to deposition of $\mathrm{Cu}$ nanoparticles on the CNT surfaces [21].

\section{CONCLUSION}

CNTs with metallic particles show conductive properties and when they are used as fillers in paints, they tend to acquire those conductive properties and such paints can be used as solar paints. Various techniques of CNT preparation with incorporation of metallic particles, functionalization techniques and dispersion techniques have been discussed in this review. Thus, the suitable technique for the paint preparation with addition of CNTs to be chosen for attaining the required properties

\section{REFERENCES}

[1] O.P Agnihotri and B.K. Gupta, Solar Selective Surfaces, John Wiley \& Sons, 1981, pp. 159-168.

[2] Matthew P. Genovese, Ian V. Lightcap and Prashant V. Kamat, Sun-Believable Solar Paint: A Transformative One-Step Approach for Designing Nanocrystalline Solar Cells, American Chemical Society, 2012, vol. 6, pp. 865-872.

[3] Jiangtao Zhu,Juncai Jia,Fung-luen Kwong and Leung Hang Ng Dickon, Synthesis of bamboo like carbon nanotubes on a copper foil by catalytic chemical vapor deposition from ethanol, Carbon, 2012, vol. 50, pp. 2504-2512.

[4] Veena Choudhary and Anju Gupta, Carbon Nanotubes - Polymer Nanocomposites, InTech Publishers, pp. 65-67, 2012.

[5] Z Jiang, R Song, W. Bi, J. Lu and T. Tang, Polypropylene as a carbon source for the synthesis of multi-walled carbon nanotubes via catalytic combustion, Carbon, 2007, vol. 45, pp. 449-458.

[6] J. Liu, Z. Jiang, H. Yu and T. Tang, Catalytic pyrolysis of polypropylene to synthesize carbon nanotubes and hydrogen through a two-stage process, Polymer Degradation and Stability, 2011, vol. 96, pp. 1711-1719.

[7] D. Pradhan and M. Sharon. Carbon nanotubes, nanofilaments and nanobeads by thermal chemical vapor deposition process. Materials Science and Engineering B, 2002, vol. 96, pp. 24-28.

[8] U. Arena, M. Mastellone, G. Camino and E. Boccaleri, An innovative process for mass production of multi-wall carbon nanotubes by means of low-cost pyrolysis of polyolefin. Polymer Degradation and Stability, 2006, vol. 91, pp. 763-768.

[9] J. Zhang, J. Du, Y. Qian and S. Xiong, Synthesis, characterization and properties of carbon nanotubes microspheres from pyrolysis of polypropylene and maleated polypropylene, Material Research Bullitein, 2010, vol. 45, pp. 15-20.

[10] C. Zhuo, B. Hall, H. Richter, Y. Levendis. Synthesis of carbon nanotubes by sequential pyrolysis and combustion of polyethylene. Carbon, 2010, vol. 48, pp. 4024-4034.

[11] J. Gong, J. Liu, Z. Jiang, J. Feng, X. Chen, L. Wang, E. Mijowsk, $\mathrm{X}$. Wen and T. Tang. Striking influence of chain structure of polyethylene on the formation of cup-stacked carbon nanotubes/carbon nanofibers under the combined catalysis of $\mathrm{CuBr}$ and NiO. Applied Catalysis B, 2014, vol. 147, pp. 592-601.

[12] Mukul Kumar and Yoshinori Ando, Chemical Vapor Deposition of Carbon Nanotubes: A Review on Growth Mechanism and Mass Production, Journal of Nanoscience and Nanotechnology, 2010, vol. 10, pp. 3739-3758.

[13] Y. Li, X. Zhang, X. Tao, J. Xu, W. Huang, J. Luo, Z. Luo, T. Li, F. Liu, Y. Bao, and H. Geise, Mass production of high-quality multiwalled carbon nanotube bundles on a $\mathrm{Ni} / \mathrm{Mo} / \mathrm{MgO}$ catalyst, Carbon, 2005, vol. 43, pp. 295-301. 
[14] G.S. Bajad, S. Tiwari and R.P. Vijayakumar, Synthesis and characterization of CNTs using polypropylene waste as precursor, Materials Science and Engineering B, 2015, vol. 194, pp. 68-77.

[15] Korneva Guzeliya, Functionalization of carbon nanotubes, A Thesis Submitted to the Faculty of Drexel University,2008

[16] Ma Peng-Cheng, Naveed A. Siddiqui, Gad Marom and Jang-Kyo Kim, Dispersion and Functionalization of Carbon Nanotubes for Polymer-Based Nanocomposites, Composites Part A: Applied Science and Manufacturing, 2010, vol. 41A: issue 10, pp. 1345-1367.

[17] Benjamin Gebhardt, Type selective functionalization of single walled carbon nanotubes, 2012

18] Yan Yan Huang and Eugene M. Terentjev, A review on "Dispersion of carbon nanotubes: mixing, sonication, stabilization and composite properties, Polymers, 2012, vol. 4:issue 1, pp. 275-295.

[19] Michael Berkei, Conductive Coatings Using Carbon Nanotubes, CHEManager Europe, 2011, vol. 10, pp. 7-8.

[20] Neetu Jha and S. Ramaprabhu, Synthesis and Thermal Conductivity of Copper Nanoparticle Decorated Multiwalled Carbon Nanotubes Based Nanofluids, Journal Physical Chemistry C, 2008, vol. 112 issue:25, pp. 9315-9319

[21] Kelly L. Stano, Rachel Chapla, Murphy Carroll, Joshua Nowak, Marian McCord and Philip Bradford, Copper-Encapsulated Vertically Aligned Carbon Nanotube Arrays, ACS Applied Materials \& Interfaces, 2013, vol. 5: issue 21, pp. 10774-10781 